\title{
SEGURANÇA DO PACIENTE NA COMPREENSÃO DE ESTUDANTES DE ENFERMAGEM DE UMA UNIVERSIDADE COMUNITÁRIA
}

\section{PATIENT SAFETY IN UNDERSTANDING NURSING STUDENTS OF A COMMUNITY UNIVERSITY}

\section{SEGURIDAD DEL PACIENTE EN COMPRENSIÓN DE ESTUDIANTES DE ENFERMERÍA DE UNA UNIVERSIDAD COMUNITARIA}

\begin{abstract}
Patrícia Bitencourt Toscani Greco ${ }^{1}$, Marina Mazzuco de Souza ${ }^{2}$, Emanuelli Ferreira Mancio da Luz ${ }^{3}$, Juliana Dal Ongaro ${ }^{4}$, Carine Santos de Almeida ${ }^{5}$, Raquel Soares Kirchhof ${ }^{6}$, Tânia Solange Bosi de Souza Magnago ${ }^{7}$
\end{abstract}

Como citar esse artigo: Greco PBT, Souza MM, Luz EFM, Ongaro JD, Almeida CS, Kirchhof RS, Magnago TSBS. Segurança do paciente na compreensão de estudantes de enfermagem de uma universidade comunitária. Rev Enferm Atenção Saúde [Internet]. 2021 [acesso em____ ]10(2):e202115. doi:10.18554/reas.v10i2.4382

\section{RESUMO}

Objetivo: Identificar a compreensão dos estudantes de Graduação em Enfermagem, de uma Universidade comunitária no Sul do Brasil, sobre a segurança do paciente. Método: Estudo transversal, com 139 estudantes do curso de enfermagem de uma Universidade Comunitária do Sul do Brasil. Foi utilizado um questionário auto preenchível com varáveis sociodemográficas, acadêmicas e o instrumento relacionadas a aspectos conceituais e atitudinais sobre erro humano e segurança do paciente. Resultados: Evidenciou-se a prevalência de estudantes com aprendizado formal sobre o tema, porém demonstraram incerteza com determinados conceitos, bem como com algumas atitudes relacionadas a segurança do paciente. Conclusão: Os estudantes demonstraram percepções favoráveis à segurança do paciente, como a importância de fortalecer discussões sobre esta temática no ambiente acadêmico.

Descritores: Enfermagem; Estudantes de Enfermagem; Educação Superior; Segurança do Paciente.

\footnotetext{
${ }^{1}$ Enfermeira. Doutora em Enfermagem. Universidade Regional Integrada do Alto Uruguai e das MissõesCampus Santiago/RS. http://orcid.org/0000-0001-6999-5470

2 Enfermeira. Mestre em Enfermagem. Universidade Regional Integrada do Alto Uruguai e das MissõesCampus Santiago/RS. http://orcid.org/0000-0002-3607-9836

${ }^{3}$ Enfermeira. Doutoranda em Enfermagem pela UFSM. Universidade Federal de Santa Maria (UFSM). Santa Maria/RS. http://orcid.org/0000-0002-7799-5232

${ }^{4}$ Enfermeira. Doutoranda em Enfermagem pela UFSM. Universidade Federal de Santa Maria (UFSM). Santa Maria/RS. http://orcid.org/0000-0003-0058-0132

${ }^{5}$ Enfermeira. Hospital Santa Casa. Alegrete/RS. http://orcid.org/0000-0001-9738-0043

${ }^{6}$ Enfermeira. Mestre em Enfermagem. Universidade Regional Integrada do Alto Uruguai e das Missões-Campus Santiago/RS. http://orcid.org/0000-0001-6025-9880

7 Enfermeira. Doutora em Enfermagem. Universidade Federal de Santa Maria. Santa Maria/RS. http://orcid.org/0000-0002-5308-1604
} 


\begin{abstract}
Objective: To identify the understanding of undergraduate nursing students at a community university in southern Brazil, about patient safety. Method: Cross-sectional study with 139 nursing students at a Community University in Southern Brazil. A self-administered questionnaire with sociodemographic and academic variables and the instrument related to conceptual and attitudinal aspects of human error and patient safety was used. Results: The prevalence of students with formal learning on the topic was evidenced; however they demonstrated uncertainty with certain concepts, as well as with some attitudes related to patient safety. Conclusion: Students demonstrated favorable perceptions of patient safety, such as the importance of strengthening discussions on this topic in the academic environment.
\end{abstract}

Descriptors: Nursing; Students, Nursing; Education, Higher; Patient Safety.

\title{
RESUMEN
}

Objetivo: identificar la comprensión de los estudiantes de pregrado de enfermería en una universidad comunitaria en el sur de Brasil, sobre la seguridad del paciente. Método: estudio transversal con 139 estudiantes de enfermería en una universidad comunitaria del sur de Brasil. Se utilizó un cuestionario autoadministrado con variables sociodemográficas y académicas y el instrumento relacionado con los aspectos conceptuales y actitudinales del error humano y la seguridad del paciente. Resultados: se evidenció la prevalencia de estudiantes con aprendizaje formal sobre el tema, sin embargo, demostraron incertidumbre con ciertos conceptos, así como con algunas actitudes relacionadas con la seguridad del paciente. Conclusión: los estudiantes demostraron percepciones favorables de la seguridad del paciente, como la importancia de fortalecer las discusiones sobre este tema en el entorno académico.

Descriptores: Enfermería; Estudiantes de Enfermería; Educación Superior; Seguridad del Paciente.

\section{INTRODUÇÃO}

A Segurança do paciente caracterizase pela redução do risco de danos desnecessários associados à assistência em saúde. ${ }^{1}$ Compreende-se que uma assistência à saúde de qualidade, com o intuito de promover a segurança do paciente, é um direito do indivíduo. Para isso, os serviços de saúde devem oferecer uma atenção efetiva e segura ao paciente em todo o processo. ${ }^{2}$

Abordar este tema é fundamental para a qualificação dos serviços e a construção de uma cultura positiva em prol da segurança do paciente. Nessa perspectiva, no Brasil, o Ministério da Saúde, instituiu o Programa Nacional de Segurança do Paciente (PNSP), em 2013, o qual pode ser considerado como marco do comprometimento com a qualidade e o cuidado seguro. $^{3}$

Neste contexto, o PNSP, articulado ao Ministério da Educação e Conselho Nacional de Educação, traz a inclusão do tema segurança do paciente no ensino técnico, superior e pós-graduação na área da saúde, no intuito de produzir, sistematizar e difundir conhecimentos sobre o tema, além da educação 
permanente dos profissionais. É fundamental que os profissionais da saúde sejam capazes de reconhecer e empregar as práticas baseadas em evidências na segurança do paciente, com o intuito de prevenir ou minimizar o impacto dos eventos adversos na saúde do paciente. ${ }^{4}$

É indispensável que os cursos de Graduação em Enfermagem abordem a segurança do paciente durante a formação acadêmica. E assim, ofereçam subsídios à ações de enfermagem a fim de promover conhecimentos e habilidades acerca de estratégias que reduzam erros aprimorem a segurança do paciente, as quais não estejam apenas voltadas para questões de conhecimento técnico, mas sim da necessidade de se trabalhar a segurança enquanto cultura organizacional e ferramenta gerencial para o enfermeiro. ${ }^{5}$

A realização deste estudo vem ao encontro de dois objetivos específicos do PNSP: produzir, sistematizar e difundir conhecimentos sobre segurança do paciente; e fomentar a inclusão do tema no ensino técnico, de graduação e pósgraduação na área da saúde. ${ }^{3}$ Sendo assim, tem-se como problema de pesquisa: Qual a compreensão de acadêmicos do curso de graduação em enfermagem sobre segurança do paciente? A hipótese deste estudo é que os estudantes de enfermagem possuem uma compreensão favorável sobre a segurança do paciente. Nesse sentido, objetivou-se identificar a compreensão dos estudantes de Graduação em Enfermagem, de uma Universidade comunitária no Sul do Brasil, sobre segurança do paciente.

\section{MÉTODO}

Estudo transversal descritivo, realizado com estudantes do curso de Graduação em Enfermagem, de uma Universidade Comunitária do Sul do Brasil. Na oportunidade da realização do estudo, estavam matriculados 165 estudantes. Considerando um cálculo amostral com erro de $5 \%$ e percentual estimado de $50 \%$, estimou-se uma amostra mínima de 117 participantes. Foram incluídos todos os estudantes matriculados no curso, e excluídos os menores de 18 anos, em afastamento (licenças e exercícios domiciliares), ou ainda trancamento ou transferência, e a acadêmica coletadora dos dados.

A coleta dos dados ocorreu entre setembro e outubro de 2016, por meio de questionário auto preenchível composto por duas partes. A primeira, contemplava variáveis sociodemográficas e acadêmicas (idade, sexo, procedência, raça e orientação formal sobre segurança do paciente). A segunda, composta por 20 questões relacionadas a aspectos conceituais (7) e atitudinais (13) sobre erro 
humano e segurança do paciente ${ }^{4}$, distribuídos em uma escala do tipo Likert de 5 pontos que varia de "concordo fortemente" até "discordo fortemente". Os aspectos conceituais consistem no conhecimento dos acadêmicos em relação à teoria, e os atitudinais referem-se à intenção ou a predisposição para a ação, que deve ser realizada. ${ }^{4}$

Para inserção dos dados foi utilizado o programa, Epi-info®, versão 6.4, com dupla digitação independente. Após a verificação de erros e inconsistências, a análise dos dados foi realizada no programa

PASW

Statistics ${ }^{\circledR}$ (PredictiveAnalytics Software, da SPSS Inc., Chicago - USA) 18.0 for Windows, por meio da estatística descritiva. Os resultados são apresentados por frequências absolutas $(\mathrm{N})$ e relativas $(\%)$.

Este estudo cumpriu as exigências contidas na Resolução 466/2012. Também recebeu autorização do Comitê de Ética em Pesquisa (COEP) sob número: 1687552, e CAAE nº 58499316.8.00005353.

\section{RESULTADOS}

Participaram do estudo 139 estudantes. As perdas contemplam dois afastamentos, 15 trancamentos e/ou transferências, oito que não foram encontrados no período de coleta dos dados, um óbito e a acadêmica coletadora da pesquisa.

Prevaleceram estudantes do sexo feminino $(\mathrm{N}=121 ; 87,1 \%)$, raça branca $(\mathrm{N}=121 ; 87,1 \%)$, com média de 23,9 anos de idade $( \pm 5,45$; mínimo $=18$ e máximo $=$ 44), provenientes do município sede da universidade $\quad(\mathrm{N}=70 ; \quad 50,4 \%)$. Os estudantes afirmam ter aprendizado formal sobre segurança do paciente $(\mathrm{N}=96$; $69,1 \%)$.

$\mathrm{Na}$ Tabela 1, está apresentada a distribuição das respostas dos estudantes sobre os aspectos conceituais sobre erro humano e segurança do paciente. 
Tabela 1 - Distribuição das respostas dos estudantes sobre os aspectos conceituais referentes ao erro humano e à segurança do paciente. Santiago, RS, Brasil, 2016. (N=139)

\begin{tabular}{|c|c|c|c|c|c|c|}
\hline \multirow{2}{*}{ Aspectos conceituais } & $\mathbf{C F}$ & C & NO & D & DF & NR \\
\hline & $\mathbf{N}(\%)$ & $\mathbf{N}(\%)$ & $\mathbf{N}(\%)$ & $\mathbf{N}(\%)$ & $\mathbf{N}(\%)$ & $\mathbf{N}(\%)$ \\
\hline $\begin{array}{l}\text { Cometer erros na área da saúde é } \\
\text { inevitável. }\end{array}$ & $19(13,8)$ & $59(42,4)$ & $7(5,0)$ & $37(26,6)$ & $17(12,2)$ & - \\
\hline $\begin{array}{l}\text { Existe uma grande diferença entre o } \\
\text { que os profissionais sabem o que é } \\
\text { certo e o que é visto no dia a dia da } \\
\text { assistência à saúde. }\end{array}$ & $44(31,7)$ & $77(55,4)$ & $12(8,6)$ & $4(2,9)$ & $1(0,7)$ & $1(0,7)$ \\
\hline $\begin{array}{l}\text { Profissionais competentes não } \\
\text { cometem erros que causam danos aos } \\
\text { pacientes. }\end{array}$ & $10(7,2)$ & $28(20,1)$ & $5(3,6)$ & $77(55,4)$ & $19(13,7)$ & - \\
\hline $\begin{array}{l}\text { Alunos comprometidos não cometem } \\
\text { erros que causam danos aos } \\
\text { pacientes. }\end{array}$ & $7(5,0)$ & $29(20,9)$ & $6(4,3)$ & $82(59,0)$ & $15(10,8)$ & \\
\hline $\begin{array}{l}\text { Na vigência de um erro, todos os } \\
\text { envolvidos (profissionais, alunos, } \\
\text { gestores, paciente e família) devem } \\
\text { discutir sua ocorrência. }\end{array}$ & $63(45,3)$ & $57(41,0)$ & $7(5,0)$ & $9(6,5)$ & $3(2,2)$ & - \\
\hline $\begin{array}{l}\text { Para a análise do erro humano é } \\
\text { importante saber quais as } \\
\text { características individuais } \\
\text { profissional que cometeu o erro. }\end{array}$ & $32(23,0)$ & $89(64,0)$ & $10(7,3)$ & $7(5,0)$ & $1(0,7)$ & - \\
\hline $\begin{array}{l}\text { Depois que um erro ocorre, uma } \\
\text { efetiva estratégia de prevenção é } \\
\text { trabalhar com maior cuidado. }\end{array}$ & $64(46,0)$ & $64(46,0)$ & $3(2,3)$ & $7(5,0)$ & $1(0,7)$ & - \\
\hline
\end{tabular}

Fonte: Dados da pesquisa, 2016.

Legenda: DF - Discordo Fortemente D - Discordo; NO - Não tenho Opinião; C - Concordo; CF - Concordo; NR- Não respondeu.

A Tabela 2 apresenta a distribuição das respostas sobre os aspectos atitudinais quanto ao erro humano e segurança do paciente. 
Tabela 2- Distribuição das respostas dos estudantes sobre os aspectos atitudinais referentes ao erro humano e à segurança do paciente. Santiago, RS, Brasil, 2016. (N=139)

\begin{tabular}{|c|c|c|c|c|c|c|}
\hline Aspectos atitudinais & $\begin{array}{c}\text { CF } \\
\mathbf{N}(\%)\end{array}$ & $\begin{array}{c}\mathrm{C} \\
\mathrm{N}(\%)\end{array}$ & $\begin{array}{c}\text { NO } \\
\mathrm{N}(\%) \\
\end{array}$ & $\begin{array}{c}\text { D } \\
\mathrm{N}(\%)\end{array}$ & $\begin{array}{c}\text { DF } \\
\mathbf{N}(\%)\end{array}$ & $\begin{array}{c}\text { NR } \\
\mathbf{N}(\%)\end{array}$ \\
\hline $\begin{array}{l}\text { Profissionais não devem tolerar trabalhar } \\
\text { em locais que não oferecem condições } \\
\text { adequadas para o cuidado prestado ao } \\
\text { paciente } \\
\text { Para implementar medidas de prevenção } \\
\text { de erros humanos, sempre se deve instituir } \\
\text { uma análise sistêmica dos fatos }\end{array}$ & $54(38,8)$ & $60(43,2)$ & $10(7,2)$ & $14(10,1)$ & $1(0,7)$ & - \\
\hline $\begin{array}{l}\text { É necessário implementar análise sistêmica } \\
\text { de } \quad \text { erros } \\
\text { da na }\end{array}$ & $43(30,9)$ & $70(50,4)$ & $10(7,2)$ & $12(8,6)$ & $4(2,9)$ & - \\
\hline $\begin{array}{l}\text { Sempre comunico } \\
\text { professor sobre a presença de condições no } \\
\text { campo de estágio que favorecem a } \\
\text { ocorrência do erro }\end{array}$ & $63(45,3)$ & $66(47,5)$ & $9(6,5)$ & $1(0,7)$ & - & - \\
\hline 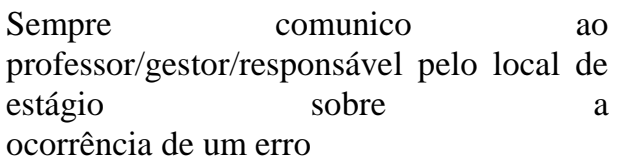 & $69(49,6)$ & $61(43,9)$ & $7(5,1)$ & $2(1,4)$ & - & - \\
\hline $\begin{array}{l}\text { Sempre comunico ao meu colega sobre a } \\
\text { ocorrência do erro }(n=138)\end{array}$ & $29(20,9)$ & $66(47,5)$ & $19(13,7)$ & $23(16,5)$ & $1(0,7)$ & $1(0,7)$ \\
\hline $\begin{array}{l}\text { Sempre comunico ao paciente e sua } \\
\text { família sobre a ocorrência do erro }\end{array}$ & $13(9,4)$ & $54(38,8)$ & $42(30,2)$ & $27(19,4)$ & $3(2,2)$ & - \\
\hline \begin{tabular}{lccr} 
Se não & \multicolumn{2}{c}{ ocorre dano } & ao \\
paciente, deve-se & analisar se & há \\
necessidade & de & relatar & a \\
ocorrência do erro ao paciente e família.
\end{tabular} & $10(7,2)$ & $53(38,1)$ & $20(14,4)$ & $50(36,0)$ & $6(4,3)$ & - \\
\hline $\begin{array}{l}\text { Os professores } \\
\text { realizam medidas corretivas com o aluno } \\
\text { para que ele não cometa novos erros }\end{array}$ & $51(36,7)$ & $63(45,3)$ & $14(10,1)$ & $8(5,8)$ & $3(2,2)$ & - \\
\hline $\begin{array}{l}\text { Sistemas para relatar } \\
\text { ocorrência dos erros fazem pouca } \\
\text { diferença na redução de futuros erros } \\
\text { Apenas os médicos podem determinar a } \\
\text { causa da ocorrência do erro }\end{array}$ & $4(2,9)$ & $22(15,8)$ & $14(10,1)$ & $79(56,8)$ & $20(14,4)$ & - \\
\hline $\begin{array}{l}\text { Sempre realizo atividades de estágio em } \\
\text { locais que }\end{array} \begin{array}{c}\text { promovem } \\
\text { boas práticas para a promoção da } \\
\text { segurança do paciente } \\
\text { Sempre }\end{array}$ & $27(19,4)$ & $50(36,0)$ & $29(20,9)$ & $27(19,4)$ & $6(4,3)$ & - \\
\hline $\begin{array}{l}\text { situações } \\
\text { melhorias, que }\end{array}$ & $11(7,9)$ & $50(36,0)$ & $39(28,1)$ & $33(23,7)$ & $6(4,3)$ & - \\
\hline
\end{tabular}

Fonte: Dados da pesquisa, 2016.

Legenda: DF - Discordo Fortemente D - Discordo; NO - Não tenho Opinião; C - Concordo; CF - Concordo; NR- Não respondeu 


\section{DISCUSSÃO}

Estudos realizados na Universidade Federal de Santa Maria $\left(\mathrm{UFSM}^{6}{ }^{6}\right.$ e Universidade Federal do Triângulo Mineiro (UFTM) ${ }^{7}$ evidenciam similaridade no que se refere à caracterização sociodemográfica dos estudantes: do sexo feminino $(86,1 \%)^{6}$ e $(89 \%)^{7}$, bem como jovens, com idade entre 18 a 22 anos $(56,1 \%)^{6} \quad(34 \%) .^{7}$ Também quanto à procedência dos estudantes ser da cidade sede da universidade. ${ }^{7}$ No entanto, difere do estudo realizado na UFSM. ${ }^{6}$

Identificou-se que os estudantes possuem aprendizado formal sobre segurança do paciente durante a Graduação em Enfermagem. Mesmo que a Universidade pesquisada não ofereça uma disciplina específica, esse tema é contemplado nos diferentes semestres e cenários de prática. Assim como, em estudo realizado na $\mathrm{UFSM}^{6}$ e na Universidade Federal de São Paulo $(\mathrm{UNIFESP})^{4}$, em que os estudantes também demonstraram percepções favoráveis sobre segurança do paciente, o que confirma que o tema tem sido abordado nos cursos de forma transversal. ${ }^{8,9}$

Em relação aos aspectos conceituais, $42,4 \%$ dos estudantes concordam que cometer erros na área da saúde é irrevogável, demonstrando a percepção de que errar é humano. Porém, talvez não tenham a compreensão de que pode ser evitado por meio de medidas preventivas. Sabe-se que é inviável a hipótese de eliminar o erro totalmente, mas há mecanismos para evitar o erro e os EA. ${ }^{8}$

Sabe-se que, compreender a relação entre riscos e características dos cuidados pode obter elementos importantes para a melhoria da assistência. Esse conhecimento é relevante para estabelecer articulações entre os serviços de saúde, desencadear ações educativas, contribuir para a redução da mortalidade, associada a EA graves e melhorar a qualidade de vida de pacientes e profissionais. $^{9}$ Neste contexto, para se obter uma assistência segura e de qualidade, o profissional deve ter prudência e comprometimento frente suas ações de cuidados ao paciente. ${ }^{10}$

Os estudantes, concordam que é importante conhecer as características individuais do profissional que cometeu o erro, o que se assemelha a outros estudos nacionais., ${ }^{4,6}$ Ao acreditar que características pessoais são importantes para avaliação do erro, contradiz-se com o modelo proposto por Reason, que esclarece a análise sistêmica do erro, o qual substitui a abordagem individual que remete à culpa. No modelo sistêmico, são discutidas e avaliadas as diversas etapas do processo de trabalho que podem apresentar falhas e culminar em erro ou incidente. ${ }^{11}$ 
No que se refere à vigência de um erro, os estudantes concordam que todos envolvidos devem discutir sua ocorrência, assim como que uma efetiva estratégia de prevenção é trabalhar com maior cuidado. Dado esse, em acordo com os resultados dos estudos da UNIFESP ${ }^{4}$ e UFSM. ${ }^{6}$ Pode ser considerado como ação de prevenção eficaz, trabalhar com cuidado, no entanto remete a uma visão voltada ao sujeito, porém, para prevenir erros humanos é necessário compreender como acontecem. ${ }^{11}$ Destaca-se que em organizações complexas, um único erro na ponta raramente é suficiente para causar dano. $^{12}$ Estudo destaca que o uso de simulação no processo ensinoaprendizagem é uma estratégia inovadora para ser incentivada, ainda, de forma complementar estudos de caso, mapa conceitual, aprendizagem baseada em problemas podem estimular o pensamento crítico-reflexivo de estudantes. ${ }^{13}$

$\mathrm{O}$ erro não deve ser visto de forma individual e sim sistematicamente, a abordagem deve preconizar os fatores sistêmicos e quais os contribuintes que acarretaram o mesmo. Quando as medidas corretivas são vistas de forma individual, destacando quem errou, promove uma falsa segurança em relação à resolução do problema. Nesse sentido, destaca-se a relevância de ações não punitivas, e sim com vistas a oportunidades de aprender com os erros, bem como discuti-los com a equipe envolvida e pacientes. ${ }^{10}$

Ressalta-se que ao prestar o cuidado tem-se um cenário complexo e dinâmico com situações adversas que podem predispor o erro. ${ }^{14}$ Assim, trabalhar com atenção é necessário, porém reconhece-se que o ritmo de trabalho acelerado, a sobrecarga, e um constante estado de alerta são características inerentes ao trabalho da enfermagem, o que muitas vezes interfere no cuidado. Estudo realizado com enfermeiros aponta que a sobrecarga de trabalho, falta de atenção do profissional, despreparo ou má-formação profissional, automatização do cuidado, prescrição ilegível, falta de profissionais, falta de planejamento e ambiente físico inadequado nos serviços de saúde são fatores que contribuem para o erro. ${ }^{15}$

Em relação aos aspectos atitudinais, os estudantes concordam que profissionais não devem tolerar trabalhar em locais que não oferecem condições adequadas para o cuidado prestado ao paciente. Este resultado converge com resultados encontrados no estudo da UNIFESP. ${ }^{4}$ Assim, percebe-se que os estudantes compreenderam que condições inadequadas no local de trabalho podem contribuir para a ocorrência de EA.

Além do ambiente de trabalho ser favorável para uma assistência segura, deve-se também considerar o compromisso 
ético dos profissionais e estudantes para com os pacientes. Pois, para ter uma cultura de segurança positiva a assistência deve ser pautada na promoção da saúde e livre de EA relacionados a imperícia, negligência ou imprudência. ${ }^{16}$

Quanto à implementar medidas de prevenção de erros, os estudantes concordam que sempre se deve instituir uma análise sistêmica de erros na área da saúde. O que se assemelha aos resultados do estudo realizado na UNIFESP $^{4}$ e na UFSM. ${ }^{6}$ A abordagem de sistemas, nesse contexto, visa identificar situações ou fatores susceptíveis a origem de erros humanos, e implementar sistemas de mudanças que irão reduzir a sua ocorrência ou minimizar seu impacto frente a assistência prestada ao paciente. ${ }^{11}$ Observar esta compreensão no estudante é relevante, pois demonstra que estes podem estar reflexivos sobre os EA, e o erro, o que poderá favorecer uma mudança do modelo de avaliação individual para um modelo ampliado e sistêmico.

Ainda, os estudantes concordaram que deve-se comunicar o professor sobre a presença de condições inadequadas no campo de estágio, as quais possam favorecer a ocorrência do erro. É importante ressaltar que a segurança do paciente é responsabilidade de todos os envolvidos nos cuidados à saúde, nesse sentido, os estudantes precisam ser parceiros neste processo. Com isso favorecer a oportunidade de aprendizado baseada em uma comunicação clara, pautada na prevenção de EA, no trabalho em equipe, na assistência centrada no paciente e em aprender com os erros é recomendado em todo o processo de processo de formação em saúde. ${ }^{19}$

Quanto às assertivas, referentes à comunicação do erro ao paciente, sua família e ao colega, os estudantes concordaram. Da mesma forma, concordam que se não ocorre dano ao paciente, deve-se analisar se há necessidade de relatar a ocorrência do erro ao paciente e família. Esses resultados corroboram com os de estudo realizado na UFSM. $^{6}$

Compreende-se que inserir o paciente e a família/cuidador como parceiros do cuidado é um desafio para os profissionais da área da saúde, visto que estes não são preparados para um planejamento na assistência em conjunto com paciente e familiar/cuidador. No entanto, para que se alcance uma assistência eficaz e de qualidade, os familiares devem estar envolvidos no seu processo de cuidado e na sua segurança, tendo o direito de serem informados, fortalecendo assim as relações equipepaciente e a eficácia do tratamento. ${ }^{17}$

Evidenciou-se que os estudantes discordaram que os sistemas para relatar o 
erro, fazem pouca diferença na redução de futuros erros. Estes dados estão alinhados aos resultados obtidos em outros estudos. ${ }^{4,6}$ Entende-se que sistemas de notificação são ferramentas que devem ser utilizadas e disseminadas nos serviços de saúde, no intuito de monitorar e avaliar as intercorrências na prestação do cuidado. Estes possibilitam as instituições o desenvolvimento de capacitações quanto a notificação, bem como auxiliam na tomada de decisão para implementação de melhorias nos processos de trabalho. É consenso mundial que o conhecimento, a avaliação e a discussão dos erros notificados fortalecem o estabelecimento de melhorias para o serviço, sendo este o principal foco dos Núcleos de Segurança do Paciente. ${ }^{11}$

No que tange a receber apoio da instituição para implementação de medidas que promovam práticas seguras, os estudantes concordaram. Entretanto, estudo realizado na UFSM, obteve-se um maior percentual de estudantes que não tinham opinião sobre o assunto. ${ }^{6}$ Assim, observa-se que a ponderação dos estudantes sobre o apoio da instituição para melhorar a prestação de cuidado ao paciente, pode ter relação com as disciplinas ofertadas pelo curso de enfermagem da referida universidade. Disciplinas essas como o Projeto de Intervenção Profissional, o estágio supervisionado e o trabalho de conclusão de curso, que estão diretamente relacionadas ao planejamento e execução de ações assistenciais, as quais podem contribuir com a segurança do paciente.

Identificou-se uma quantidade significativa de resposta "não tenho opinião", o que não possibilita identificar se apresentam ou não entendimento sobre o tema. Frente ao exposto, para que haja um cuidado seguro, torna-se imprescindível a construção do conhecimento por parte dos estudantes acerca de conceitos e atitudes para uma cultura de segurança do paciente positiva. Ademais, para que o cuidado seja seguro, necessita-se que profissionais e serviços compartilhem de práticas e condutas que contribuam para a promoção do cuidado e redução do dano. ${ }^{18}$ Para isso, princípios relacionados a segurança do paciente são inquestionáveis na formação do acadêmico de enfermagem, aliados a uma assistência ética, resolutiva e que assegure a qualidade. $^{19}$

\section{CONCLUSÃO}

Prevaleceram os estudantes que concordam que cometer erros na área da saúde é inevitável bem como, conhecer as características individuais desse profissional é importante, porém discordaram que alunos e profissionais 
comprometidos não cometem erros. No que tange aos aspectos atitudinais os estudantes concordaram que profissionais não devem tolerar trabalhar em locais que não oferecem condições adequadas para o cuidado prestado ao paciente. Em relação à comunicação do erro, concordaram que o fariam, e que os sistemas para relatar as situações de erro fazem diferença na prevenção de futuras situações.

O estudo possibilitou estudantes e docentes perceberem a importância de fortalecer discussões sobre a segurança do paciente no ambiente acadêmico. Nesse sentido, expõem-se como limitação do estudo, referente ao instrumento, o qual ainda não possibilita a análise de níveis de compreensão, sobre os conhecimentos e atitudes dos estudantes, o que não viabilizou análise com correlações, ou associações. Para isso, é relevante que novos estudos com o instrumento possam estabelecer escores e pontos de corte para as dimensões avaliadas. Ainda, sugere-se que seja realizada análise multivariada dos itens, a fim de verificar a validade dimensional, bem como a avaliação da consistência interna do instrumento. Assim, torna-se necessário considerar os resultados em sua singularidade, uma vez que retrata a realidade particular desses estudantes.

Todavia, faz-se necessário o desenvolvimento de novos estudos, sobre a temática, entre os estudantes da área de saúde, visto a importância da atuação multiprofissional no âmbito da promoção da segurança do paciente. Ainda, recomenda-se que a temática seja incluída, de forma transversal, nas disciplinas já existentes dos cursos da área da saúde, contribuindo para a formação dos futuros profissionais, subsidiando-os para uma prática crítica, criativa e segura.

\section{REFERÊNCIAS}

1. WHO. World Health

Organization. Marco conceptual de la clasificación internacional para la seguridad del paciente. Versión 1.1. Informe técnico definitivo. Geneva: WHO; 2009.

2. Anvisa. Agência Nacional De Vigilância Sanitária. Assistência Segura: Uma Reflexão Teórica Aplicada à Prática. Brasília; 2013.

3. Ministério da Saúde (Brasil). Portaria ${ }^{\circ} 529$ de $1^{\circ}$ de abril de 2013. Institui o Programa Nacional de Segurança do Paciente (PNSP). D. O. U., Brasília, 2013.

4. Yoshikawa JM, Sousa BEC, Peterlini MAS, Kusahara DM, Pedreira MLG, Avelar AFM. Compreensão de alunos de cursos de graduação em enfermagem e medicina sobre segurança do paciente. Acta Paul. Enferm. 2013; 26: 21-9.

5. Bogarin DF, Zanetti ACB, Brito MFP, Machado JP, Gabriel CS, Bernardes A. Segurança do paciente: conhecimento de alunos de graduação em enfermagem. Cogitare Enfermagem. Jul/Set 2014; 19:491-7.

6. Cauduro GMR, Magnago TSBS, Andolhe R, Lanes TC, Dal Ongaro J.

Segurança do paciente na compreensão de 
estudantes da área da saúde. Rev Gaúcha Enferm. 2017;38(2):e64818.

$7 . \quad$ Nardelli GG, Gaudenci EM, Garcia BB, Carleto CT, Gontijo LM, Pedrosa LAK. Perfil dos alunos ingressantes dos cursos da área da saúde de uma universidade federal. Revista de enfermagem e atenção em saúde. 2013; 2(1):3-12.

8. Fernandes LGG, Tourinho FSV, Souza NL, Menezes RMP. Contribuição de James Reason para a segurança do paciente: reflexão para a prática de enfermagem. Rev enferm UFPE. Jul 2014; 8(supl. 1):2507-12.

9. Oliveira RM, Leitão IMTA, Silva L MS, Figueiredo SV, Sampaio RL, Gondim MM. Estratégias para promover segurança do paciente. Escola Anna Nery. Jan./Mar 2014; 18(1): 122-29.

10. Eberle CC, Silva APSS.

Compreensão de estudantes de enfermagem sobre a segurança do paciente. Revista Baiana de Enfermagem. Out/dez 2016; 30(4):1-9.

11. Brasil, Ministério da Saúde. Agência Nacional de Vigilância Sanitária. Documento de referência para o Programa Nacional de Segurança do Paciente.

Brasília, 2014.

12. Wachter RM. Compreendendo a segurança do paciente. Artmed, $2^{\mathrm{a}}$ edição. Porto Alegre (RS): 2013. Disponível em: https://books.google.com.br/books?hl=ptBR\&lr=\&id=LWQ3AgAAQBAJ\&oi=fnd $\& p g=P R 3 \&$ ots $=$ sACrbheWzf\&sig $=\mathrm{JeUdR}$ XnbLPZmCukawGAtmA9f72M\&redir_es $\mathrm{c}=\mathrm{y} \# \mathrm{v}=$ onepage $\& \mathrm{q} \& \mathrm{f}=$ false.

13. Lopes MNA, Beccaria LM, Silva LB, Cesarino CB, Faria JIL. Segurança do paciente: desenvolvimento do tema em cursos de graduação em enfermagem. Enfermagem Brasil. 2018;17(3):208-217. 14. Rosa RT, Gehlen MH, Ilha S, Pereira FW, Cassola T, Backes DS. Segurança do paciente na práxis do cuidado de enfermagem: percepção de enfermeiros. Ciencia y enfermeria. 2015; XXI(3):37-47.
15. Dias JD, Mekaro KS, Tibes CMS, Zem-Mascarenhas SH. Compreensão de enfermeiros sobre segurança do paciente e erros de medicação. Revista Mineira de Enfermagem. Out/dez 2014;18(4):866-873. 16. Ilha P, Radünz V, Tourinho FSV, Marinho MM. Segurança do paciente na percepção de acadêmicos de enfermagem. Cogitare Enferm. 2016; 21(especial):1-10. 17. Fonseca AS, Peterlini FL, Costa DA. Segurança do Paciente. São Paulo (SP): Martinari; 2014.

18. Rede Brasileira de Enfermagem e Segurança do Paciente (Brasil). Rede Brasileira de Enfermagem e Segurança do Paciente: Estratégias para a segurança do paciente: manual para profissionais da saúde. Porto Alegre. EDIPUCRS; 2013. 19. Calazans MSC et al. Segurança do paciente entre estudantes de enfermagem: uma revisão integrativa. Revista Enfermagem Atual In Derme. 2020; 94(32):1-7.

RECEBIDO: 01/04/2020

APROVADO: 07/06/2021

PUBLICADO: 09/2021 